\title{
THE STRATEGIC HUNT FOR MOBILE CONSUMERS THROUGH M-COMMERCE
}

\author{
David B. Scibelli, Robert Morris University, davidscibelli@yahoo.com \\ Brian Stevens, Robert Morris University, brianstevens13@gmail.com
}

\begin{abstract}
This paper's perspective is through the lens of technology advancements in data warehousing, mobile devices, technology acceptance and target advertising in creating an information system adaptive to specialized marketing to consumers based on their observed interests and behaviors. Thereby, the research and analysis approach of this study is grounded through scholarly journals and study of technology advancements allowing for the development of these systems. A deeper dive into this research examines the perceptions of consumers' acceptance of M-Commerce and the steps commercial businesses need to consider regarding the solicitation of their customers and how they are opted into target ad campaigns.
\end{abstract}

Keywords: Mobile Technology, M-Commerce, TAM, Mobile-Ads, Consumer Privacy

\section{INTRODUCTION}

The focus of this paper explores the systems technology, theory and business claim behind changing landscapeembracing M-Commerce as a viable industry driving force behind many business partnerships. These business agreements are made in the spirit of collecting and tracking consumer behaviors in order to deliver customized advertisements and marketing techniques to personal mobile devices users. The analysis of this research examines the progression of technology behind the data collection capabilities, as well as the adoption of personal devices as an integral tool used in their daily lives. Furthermore, a wider reach into the consumer acceptance of these products and willingness to embrace personalized ads. In addition, this paper will look at the strategic alliances and data collection techniques used by corporations to collect from their customers' personal information for product development and profit. The notion which underlies this paper looks at the tact companies take in to recruiting their adaptors of personalized M-Commerce ads.

\section{Data Warehousing: Changing the Landscape}

Technology has enabled companies to collect and decipher consumer information through data warehousing and data mining techniques. Considering the commodity level pricing and capability of the processors, storage and high bandwidth networks, companies have developed the capability to store Petabytes of consumer information coining in the term "Big Data".

\section{Convergence of "Personal" and "Devices"}

The development of sophisticated mobile phone technology and personal devices has brought endless possibilities to the hands of users. These tools have extended our capabilities and are now seen as a necessity for many. As adopters of this technology, we now live within the framework it has provided for us [13]. The framework to which we behold ourselves to is our mobile phone and the applications and services that connect us to our world and lifestyle. Given the landscape of this notion, as illustrated in Figure 1, we have become entrapped in layers of technology and conveniences. 


\section{Issues in Information Systems}

Volume 13, Issue 1, pp. 144-150, 2012

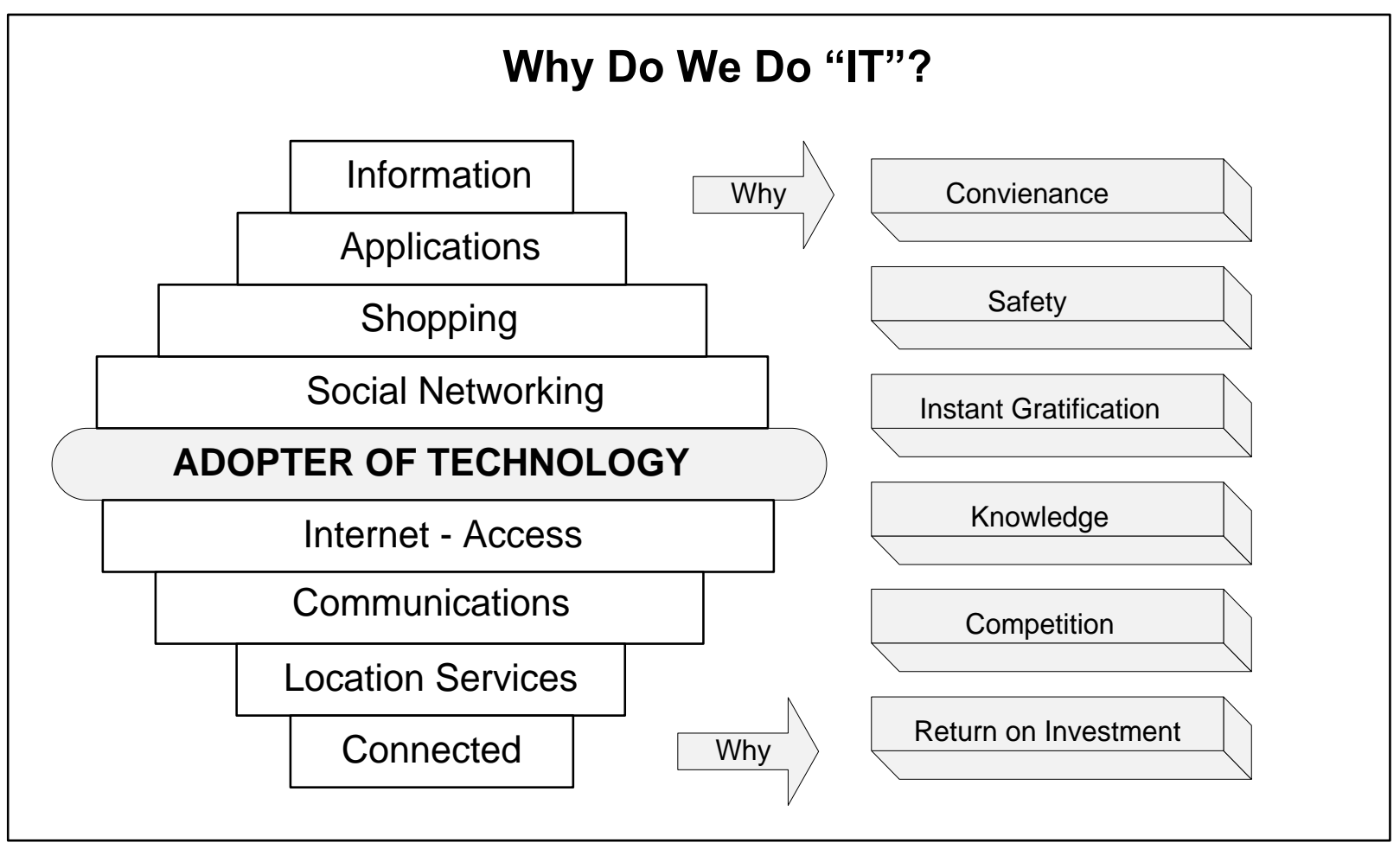

Figure 1 Consumers are embedded-in Mobile Technology

Further investigation into why consumers agree to and adopt technology into their lives is grounded in the Technology Acceptance Model (TAM). This model establishes the rationale in which consumers may embrace such technology and to why they are willing to accept the trade-offs as a user.

\section{M-Commerce - Personalized Ads}

M-Commerce is an adaptation of e-commerce, thus utilizing personal mobile devices as the medium [1]. As predicted, consumers have embraced the ease of commerce through the use of their mobile phones. The concept of target advertizing allows for specific ads to be geared to the customers, based on their interests, activities and history. With this information, commercial businesses could provide customized products and services directly to these customers.

Some measures to which companies could acquire customer information in the spirit of M-Commerce, are through the use of mobile devices including mobile phone applications, social networks, online shopping, Internet keyword searches and the use of Location Based Services. These features and services provide to the consumer great benefit and ease, however many of the products are a conduit to capturing customer information to provide them better products. Given the marketing pursuit of consumers' information, Figure 2 demonstrates the many levels of information interest which surrounds mobile device users. This representation signifies the consumer entanglement of utilizing the capabilities of the technology at their hands. Companies have positioned themselves to collect and share this information with their business partners to further provide better services to the consumer and create new products. 


\section{Issues in Information Systems}

Volume 13, Issue 1, pp. 144-150, 2012

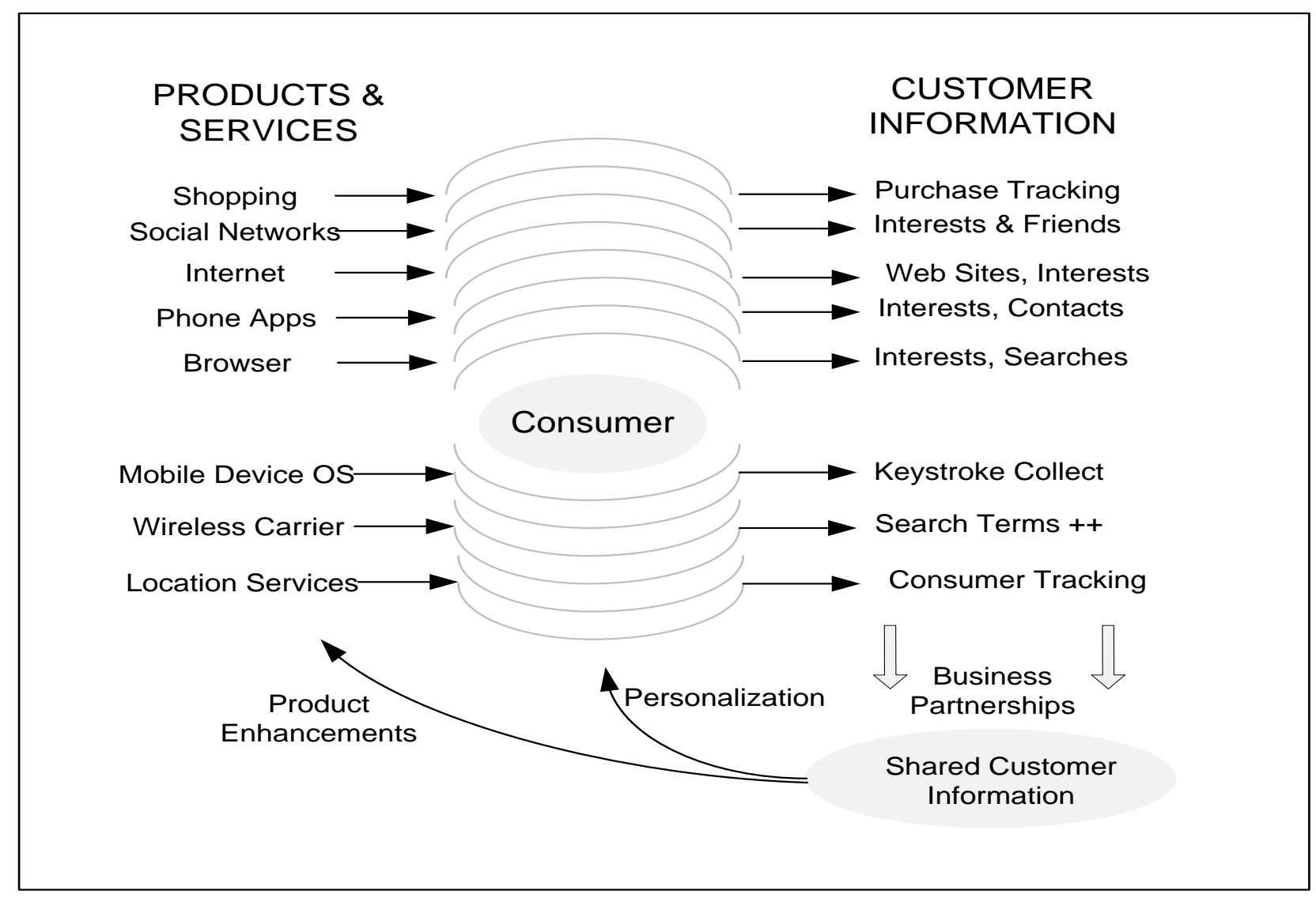

Figure 2 Consumer behaviors creating big data information aggregate.

\section{Business Delivery of Specialized Ads}

How do companies utilize this consumer information and deliver customized advertizing back to the customers? Considering the vast aggregate of consumer information collected through the use of mobile device technology platforms, is there awareness by the consumer to the levels to which their interests or activities could be played back to them; in the form of personalized ads on their mobile devices or other means? In the case of a young teenager who was receiving ads regarding baby products, which were unsolicited, her pregnancy was still a private matter, unknowing to her family [9]. Was there an opt-in choice made by the consumer regarding the use of her buying habits, to generate future ads in return? Certainly in the depths of the product agreements and store loyalty and credit card Terms of Service, the opt-in for target advertizing clause was part of the agreement. However, how receptive will consumers be regarding ads based in their current location, Facebook profile and a synopsis of their latest browser search terms? One could believe that this approach may be uncomfortable for the typical consumer.

\section{Consumer Acceptance of Specialized Ads}

Some companies are trying different approaches to connect with their customers, thereby not revealing all of what they know about them in order to not invoke any concern or surprise to their customer base [9]. Would there be an uprising by consumers if they realized the amount of personal information that is available about them, information that is shared between companies and business partners? Having a near silent opt-in agreement to have access to certain products or services could be a reasonable trade-off for many consumers as well. Research that may be 


\section{Issues in Information Systems}

Volume 13, Issue 1, pp. 144-150, 2012

noteworthy of studying regarding these trade-offs dwells within what consumers are willing to give of themselves in order to use technology products. Furthermore, will companies maintain their trustful relationships with their customers and tread lightly with their in depth knowledge of the customers?

\section{LITERATURE REVIEW}

\section{Problem Exploration}

The literary focus of this study explores the opt-in collection of consumer information through the use of mobile devices, with respect to the delivery of specialized target advertizing of products and services back to the customers. As consumers are targeted with a scheme of rich and invasive ads, will that experience be positive for customers, or will customers be uncomfortable with this marketing approach? Noteworthy to mention, often times a hidden opt-in agreement is what many consumers blindly accept. This provides a valid conduit that enables companies to collect personal information. Even simple survey results have demonstrated - at a high level - that consumers do not like personal information collected and used for target advertising [17]. With corporations using near silent data collection techniques, how will companies utilize this information without enraging the unsuspecting consumer?

\section{Data Warehousing Changed the Landscape}

The capability of technology in storing and managing data has significantly increased in line with IT advancements over time as substantiated through Moore's Law [8]. The costs of hardware, software and virtualization, conceptualized as cloud computing, has created data mining platforms which provides proficiency in collecting big data information rendering it useful for mining purposes [14].

There has been a continuous battle for consumer information driven by the marketing organizations within many companies [6]. Considering that technology has brought the business community and customers to a marketplace where personal information is also as evident in many of the social networking applications. In addition, the use of mobile technology has created an opportunity for companies to further reach for consumer information. Verizon Wireless has been an open contender for this information, and they have clearly stated their intent to use and share customers' personal interests to develop products [4]. Customers were automatically opted-in to sharing their information, although there was a process that Verizon users could reconfigure their privacy settings to opt-out of the program.

As the technology playing field has progressed into an open market for consumer data, have consumer perspectives adjusted to greater tolerances regarding the information they provide to use the technology?

\section{Business Delivery of Specialized Ads}

In the efforts of not losing customers, what is the approach that companies would take to assure "buy-in" of target advertizing from consumers? The ads need to be aligned with the customers' predilections with ease of user interaction and attract their interest [15]. Consumers do not favor mobile targeted ads unless they provided approval. Advertisements having an unfavorable solicitation imply behavioral changes in the consumer which may alter their relationship with the technology and services [18]. Based on the Theory of Reasoned Action (TRA), the ideological behaviors and attitudes of individuals could result in changes in what may have been perceived as habitual acceptance of using technology to a hardened change of behavior [7]. The impact of consumers turning against the extensive sharing of their personal information could render the data mining component for target advertising to empty databases. 


\section{Issues in Information Systems \\ Volume 13, Issue 1, pp. 144-150, 2012}

Although if businesses could provide a consumer friendly advertising approach to their customers - such as allowing them an opt-in choice and the ability to manage or control the ads they receive, mobile advertising techniques could be more acceptable to both business and consumer [12].

\section{Consumer Acceptance}

Over the past two decades, the Technology Acceptance Model, or TAM [3], has been used as the main theme of analysis to describe how willing individuals are to bring new forms of technology into their lives. The original version of the model was used to analyze a workplace e-mail system, examining the users Perceived Usefulness $(P U)$ and Perceived Ease of Use (PEU) of the program. Later adoptions of the model added emotional factors like Attitude Toward Use (AT) and Intent to Use (IU).

The model has been adapted several times from to incorporate the usage internet-based software innovations such as the World Wide Web [16], social media [2] and commercial services including online banking [11] and mobile devices [19].

Wang, Tsai, and Lu summed up their study on mobile devices by saying that "the communication effects of mobile technology are determined by the behavioral intentions of users [19].

Goodhue and Thompson [5] created the Task-Technology Fit (TTF) model to work alongside with TAM. TTF implies that the adaption of technology is dependent on how well the new technology fits the task. TAM and TTF have also been used together to predict the online shopping habits of consumers [10].

The TAM and TTF models provide a proven method for how to conduct research in a quickly-changing technology market. Early adopters are key subjects to study in these scenarios, and especially in this case, where aggressively adopting the newest model of mobile communication may potentially leave a consumer's information open and vulnerable to companies looking to exploit the individual's wants, needs, and desires.

An example of how to research the previously mentioned scenarios follows in the next section.

\section{PROPOSED RESEARCH}

The levels of acceptance outlined above can be analyzed using the data collection methods described when following the TAM and TTF models. Several TAM surveys have been adapted using mobile technology. After selecting the most suitable version, the survey will need to be adjusted to fit the research methodology and include TTF factors, which like the TAM questions, may be adopted from prior research endeavors.

The newly adopted survey will require pilot testing to ensure that any changing in wording has not deviated from the purpose of the original TAM and/or TTF surveys, and should include several open-ended qualitative questions to gauge awareness and acceptance of this phenomenon. This new survey can then be disseminated on a large-scale basis, for data collection and analysis.

\section{CONCLUSIONS}

After reviewing the literature on this subject, we must raise the question: Are consumers aware? With the plethora of apps, widgets, and other features available on ever-emerging mobile technology, the consuming public may not be aware of how many of their habits and tendencies are being tracked. 


\section{Issues in Information Systems}

Volume 13, Issue 1, pp. 144-150, 2012

In light of this, we feel many of these features should be offered in an Opt-In, rather than Opt-Out method. Consumers who desire the information would be able to subscribe to mailing/receiving lists and would be separated from those who choose not to receive those messages.

In addition to the research methodology outlined above, there can be further study on why M-Commerce consumers allow themselves to be put in this situation, namely why they do or do not investigate the 'opt-out' features offered on some of the very apps and widgets they are using.

On the other hand, how companies implement this new technology and not frighten the consumer base with how a company knows so much about their personal habits could also be studied. The fine line walked by these companies, and how they do not overstep boundaries and believe this would be a worthwhile topic to pursue.

Finally, while examining the corporate side of this equation, we have discussed using the TAM and TTF models as a means of researching consumers for educational research. We ask the question: Are these models working for Marketing Research? For the amount of knowledge companies are gaining of their customers, it may be worthwhile to compare and contrast the methods private business uses to predict consumer behavior against scientific researchers and their preferred methods.

\section{REFERENCES}

1. Clarke, I. (2001). Emerging Value Propositions for M-commerce. Journal of Business Strategies, 18(2), 41 57.

2. Curran, J. M., \& Lennon, R. (2011, November 1). Participating in the Conversation: Exploring Usage of Social Media Networking Sites. Academy of Marketing Studies Journal, 15(Special Issue 1), 21-38.

3. Davis, F. (1989). Perceived Usefulness, Perceived Ease of Use, and User Acceptance of Information Technology. MIS Quaterly, 13(3), 319-340

4. Flacy, M. (2011, October 14). Verizon Wireless starts tracking and sharing sites that users visit. Technology News, Product Reviews, Software Downloads | Digital Trends. Retrieved October 17, 2011, from http://www.digitaltrends.com/mobile/verizon-wireless-starts-tracking-and-sharing-sites-that-users-visit

5. Goodhue, D. \& Thompson, R. (1995). Task-technology fit and individual performance. MIS Quarterly, 19(2), 213-236.

6. Hagel, J., \& Rayport, J. F. (1997). The Coming Battle for Customer Information. Harvard Business Review, January-February 1997(97104), 5 - 11.

7. Hale, J. L., HouseHolder, B. J., \& Greene, K. L. (2002). 14, The Theory of Reasoned Action. The Persuasion Handbook Developments in Theory and Practice (pp. 250 - 286). Thousand Oaks: Sage Publications Inc.

8. Hill, J., Horton, M., Kling, R., \& Krishnamurthy, L. (2004). The Platforms Enabling Wireless Sensor Networks. Communications of the ACM, 47(6), 41 - 46.

9. Hill, K. (2012). How Target Figured Out A Teen Girl Was Pregnant Before Her Father Did - Forbes. Information for the World's Business Leaders - Forbes.com. Retrieved April 4, 2012.

10. Klopping, I. M., \& McKinney, E. (2004, Spring). Extending the Technology Acceptance Model and the TaskTechnology Fit Model to Consumer E-Commerce. Information Technology, Learning, and Performance Journal, 22(1), 35-47.

11. Kolodinsky, J., Hogarth, J., \& Hilgert, M. (2004). The adoption of electronic technologies by U.S. consumers. The International Journal of Bank Marketing, 22(4/5), 238-259.

12. Leppäniemi, M., \& Karjaluoto*, H. (2005). Factors influencing consumers' willingness to accept mobile advertising: a conceptual model. International Journal of Mobile Communications, 3(3), 197 - 213. Retrieved March 10, 2012, from http://www.itu.dk/people/rkva/2011-Spring-EB22/readings/Lepppaniemi-2005ConceptualModelofMobileAdvertiisingAcceptance.pdf

13. McLuhan, M. (1967). The medium is the message. New York: Bantam.

14. Mell, P., \& Grance, T. (2011). The NIST Definition of. Special Publication 800-145 (Draft), 800(145), 1 - 7. 


\section{Issues in Information Systems}

Volume 13, Issue 1, pp. 144-150, 2012

15. Mir, I. (2011). Consumer Attitude towards M-Advertizing Acceptance: A Cross-Sectional Study. Journal of Internet Banking and Commerce, 16(1), 1 - 18. Retrieved April 9, 2012, from http://www.arraydev.com/commerce/jibc/2011-04/ImranAnwar.pdf

16. Moon, J., \& Kim, Y. (2001). Extending the TAM for a World-Wide-Web context. Information \& Management, 217-230.

17. Purcell, K., Brenner, J., \& Rainie, L. (2012). Pew Internet \& American Life Project. Pew Research Center's Internet \& American Life Project. Retrieved April 10, 2012, from http://pewinternet.org/Reports/2012/SearchEngine-Use-2012/Summary-of-findings.aspx

18. Tsang, M. M., Ho, S., \& Liang, T. (2004). Consumer Attitudes Toward Mobile Advertising: An Empirical Study. International Journal of Electronic Commerce, 8(3 / Spring), 65 - 78. Retrieved April 8, 2012, from http://mesharpe.metapress.com/app/home/contribution.asp?referrer=parent\&backto=issue, 5,$8 ;$ journal,33,47;link ingpublicationresults, $1: 106045,1$

19. Wang, C., Tsai, C., \& Lu, M. (2011). Using the Technology Acceptance Model to analyze ease of use of a mobile communication system. Social Behavior and Personality, Volume 39, No. 1, pp. 65-70. 